\title{
PROBLEMA DE CONTACTO PARA UN SISTEMA DEL TIPO MINDLIN-TIMOSHENKO CON DISIPACIÓN INTERNA
}

\author{
Alfonso Perez ${ }^{1}$, Victor Tarazona ${ }^{2}$, Marco Rubio $^{3}$, \\ Amelia Villanueva ${ }^{4}$, Zoraida Huaman ${ }^{5}$ \\ Victoriano Yauri Luque ${ }^{6}$, Andrés Guardia Cayo ${ }^{7}$.
}

Resumen: En este trabajo consideramos el modelo dinámico unidimensional Mindlin-Timoshenko para vigas. Estudiaremos la existencia de soluciones para un problema de contacto asociado con el sistema Mindlin- Timoshenko. También analizaremos como su energía decae exponencialmente a cero cuando el tiempo va al infinito. El sistema planteado es como sigue:

$$
\left.\mid \begin{array}{c}
u^{\prime \prime}-u_{x x}+u+v_{x}+\alpha v^{\prime}=f \text { en } Q . \quad \text { Ecuación de Mindlin-Timoshenko } \\
v^{\prime \prime}-\left(u+v_{x}\right)_{x}=g \text { en } Q \\
u(0, \cdot)=v(0, \cdot)=0 \text { sobre }(0, T) \\
u_{x}(\ell, \cdot)+\gamma u^{\prime}(\ell, \cdot)=0 \text { sobre }(0, T) \\
u(\ell, \cdot)+v_{x}(\ell, \cdot) \geq 0, v(\ell, \cdot) \geq \phi(\ell) \text { sobre }(0, T) \\
{\left[u(\ell, \cdot)+v_{x}(\ell, \cdot)\right][v(\ell, \cdot)-\phi(\ell)]=0 \text { sobre }(0, T)} \\
u(\cdot, 0)=u_{0} ; u^{\prime}(\cdot, 0)=u_{1} \\
v(\cdot, 0)=v_{0} ; v^{\prime}(\cdot, 0)=v_{1}
\end{array}\right\} \text { Condiciones iniciales }
$$

Donde $\alpha$ y $\gamma$ son reales positivos y $Q=(0, l) \times(0, T)$ con $T>0$ real.

Palabras clave: Mindlin-Timoshenko. Decaimiento exponencial. Comportamiento asintótico.

\section{PROBLEM OF CONTACT FOR A SYSTEM OF THE TYPE MINDLIN-TIMOSHENKO WITH INTERNAL DISSIPATION}

Abstract: In this work we consider the one-dimensional dynamic model MindlinTimoshenko Beam. We will study the existence of soluciones for a contact problem associated with the Mindlin-Timoshenko system.

Also analyze how energy decays exponentially to zero as time goes to infinity.

The proposed system is as follows:

$$
\begin{aligned}
& u^{\prime \prime}-u_{x x}+u+v_{x}+\alpha v^{\prime}=f \text { en } Q \text {. Equation of Mindlin-Timoshenko } \\
& v^{\prime \prime}-\left(u+v_{x}\right)_{x}=g \text { en } Q \\
& \left.\begin{array}{l}
u(0, \cdot)=v(0, \cdot)=0 \text { sobre }(0, T) \\
u_{x}(\ell, \cdot)+\gamma u^{\prime}(\ell, \cdot)=0 \text { sobre }(0, T)
\end{array}\right\} \text { Condition of boundary } \\
& \left.\begin{array}{l}
u(\ell, \cdot)+v_{x}(\ell, \cdot) \geq 0, v(\ell, \cdot) \geq \phi(\ell) \text { sobre }(0, T) \\
{\left[u(\ell, \cdot)+v_{x}(\ell, \cdot)\right][v(\ell, \cdot)-\phi(\ell)]=0 \text { sobre }(0, T)}
\end{array}\right\} \text { Condition of contact } \\
& \left.\begin{array}{l}
u(\cdot, 0)=u_{0} ; u^{\prime}(\cdot, 0)=u_{1} \\
v(\cdot, 0)=v_{0} ; v^{\prime}(\cdot, 0)=v_{1}
\end{array}\right\} \text { Initial conditions }
\end{aligned}
$$

Where $\alpha>0, \gamma>0$ are positive real and $Q=(0, l) \times(0, T)$ with $T>0$ real.

Keywords: Mindlin-Timoshenko. Exponencial decay. Asymptotic behavior.

\footnotetext{
${ }^{1}$ UNMSM, Facultad de Ciencias Matemáticas, e-mail: apersal@hotmail.com

${ }^{2}$ UNMSM, Facultad de Ciencias Matemáticas, e-mail: vtarazonam@unmsm.edu.pe

${ }^{3}$ UNMSM, Facultad de Ciencias Matemáticas, e-mail: mrubiog@unmsm.edu.pe

${ }^{4}$ UNMSM, Facultad de Ciencias Matemáticas, e-mail: avillanuevay@unmsm.edu.pe

${ }^{5}$ UNMSM, Facultad de Ciencias Matemáticas, e-mail: zhuamang@unmsm.edu.pe

${ }^{6}$ UNMSM, Facultad de Ciencias Matemáticas, e-mail: victoriano_yauri@hotmail.com

${ }^{7}$ UNMSM, Facultad de Ciencias Matemáticas, e-mail: agcbayo@yahoo.es
} 


\section{Introducción}

Un modelo matemático físicamente bastante completo y ampliamente utilizado para describir la vibración transversal de vigas es el sistema de tipo Mindlin-Timoshenko.

La variable $u=u(x, t)$ representa el ángulo de rotación y $v=v(x, t)$ el desplazamiento vertical al tiempo $t$ de la sección transversal ubicada a $x$ unidades de un extremo.

La expresión $\sigma=u(l, \cdot)+v_{x}(l, \cdot)$ es el tensor de tensiones en la frontera y $\Phi(l)$ es la posición del obstaculo. de esta manera, $d=v(l, \cdot)-\Phi(l)$ es la distancia del cuerpo al obstáculo. Así, cuando la distancia $d$ es positiva no hay contacto $(\sigma=0)$.

Cuando no hay distancia $(d=0)$, el tensor de tensión es positivo. Sin embargo, tenemos $\sigma d=0$, para todo tiempo $t$.

El problema de equilibrio general de un cuerpo elástico lineal en contacto con un obstáculo rígido fue formulado y desarrollado ampliamente por Signorini $[9,10]$ y en mérito a ello el problema tomo el nombre de Signorini.

En el libro de Duvaut-Lions [4], se trata de las desigualdades variacionales en mecánica y física. Este libro muestra muchos problemas de contacto envolviendo desigualdades variacionales. En [3], Davaut extendió el resultado del problema de Signorini a materiales visco-elasticos lineales. Kim [7], estudió el problema de contacto con obstáculo rígido en la ecuación de la onda; Elliot-Copeti [1] y Elliot-Qi [11] analizarón el problema de contacto unidimensional en termoelásticidad.

En relación con el comportamiento asintótico de las soluciones, podemos citar a Muñoz Rivera-Olivera [8], quienes probaron que las soluciones del problema de Signorini en termoelasticidad decae exponencialmente; Nakao-Muñoz Rivera [12], muestran el decaimiento polinomial de soluciones para el problema de contacto asociado con el sistema termoviscoelástico y finalmente en Nuñpz Rivera-Portillo [6,13], prueban el decaimiento exponencial de soluciones del problema de contacto para materiales viscoelásticos.

En el presente trabajo demostraremos primeramente la existencia y unicidad de soluciones al problema de Mindlin-Timoshenko asociado a un problema de contacto mediante el método de Faedo-Galerkin usando el problema penalizado asociado al sistema. En una segunda fase demostraremos que la energía asociada al sistema planteado decae exponencialmente usando el método de la energía.

\section{Problema Penalizado}

Para obtener la solución del sistema (1) primeramente consideramos para cada $0<\varepsilon<1$, el sistema penalizado siguiente:

$$
\mid \begin{aligned}
& u_{\varepsilon}^{\prime \prime}-u_{\varepsilon x x}+u_{v_{\varepsilon}^{\prime}}+v_{\varepsilon x}+\alpha v_{\varepsilon}^{\prime}=f \text { en } Q \\
& v_{\varepsilon}^{\prime \prime}-\left(u_{\varepsilon}+v_{\varepsilon x}\right)_{x}=g \text { en } Q \\
& u_{\varepsilon}(0, \cdot)=v_{\varepsilon}(0, \cdot)=0 \text { sobre }(0, T) \\
& u_{\varepsilon x}(\ell, \cdot)+\gamma u_{\varepsilon}^{\prime}(\ell, \cdot)=0 \text { sobre }(0, T) \\
& u_{\varepsilon}(\ell, \cdot)+v_{\varepsilon x}(\ell, \cdot)-\frac{1}{\varepsilon}\left[v_{\varepsilon}(\ell, \cdot)-\phi(\ell)\right]^{-}+\varepsilon v_{\varepsilon}^{\prime}(\ell, \cdot)=0 \\
& u_{\varepsilon}(\cdot, 0)=u_{0}, u_{\varepsilon}^{\prime}(\cdot, 0)=u_{1} \text { en }(0, \ell) \\
& v_{\varepsilon}(\cdot, 0)=v_{0}, v_{\varepsilon}^{\prime}(\cdot, 0)=v_{1} \text { en }(0, \ell)
\end{aligned}
$$

donde $\xi^{-}=-\operatorname{mín}\{0, \xi\}$. Antes de analizar el sistema (2) fijaremos las siguientes notaciones.

Sea el espacio de Hilbert $V=\left\{z \in H^{1}(0, \ell) ; z(0)=0\right.$ sobre $\left.(0, T)\right\}$ Equipado con el producto interno y norma dada, respectivamente por

$$
((u, v))=\left(u_{x}, v_{x}\right)_{L^{2}(0, \ell)}=\left(u_{x}, v_{x}\right)_{2} \quad \mathrm{y} \quad\|u\|=\left|u_{x}\right|_{L^{2}(0, \ell)}=\left|u_{x}\right|_{2}
$$

Consideremos el conjunto convexo $\mathbb{K}=\{w \in V ; w(\ell) \geq \phi(\ell)$ sobre $(0, T)\}$. En este trabajo supondremos

$$
v_{0}(\ell)=\phi(\ell)
$$

Esta condición significa que la posición inicial de la viga está en contacto con el obstáculo rígido. La existencia y unicidad de la solución para el problema (2) están garantizados por el siguiente resultado: 
Teorema 2.1 Sea $\left(u_{0}, u_{1}, v_{0}, v_{1}\right) \in\left[V \cap H^{2}(0, \ell)\right] \times V$ con $v_{0}$ satisfaciendo (3) $y(f, g, \phi) \in$ $\left[H^{1}\left(0, T, L^{2}(0, \ell)\right)\right]^{2} \times C^{\infty}([0, \ell])$. Entonces, para cada $\varepsilon>0$, existe un único par de funciones $\left(u_{\varepsilon}, v_{\varepsilon}\right)$ satisfaciendo:

$$
\begin{aligned}
& \left(u_{\varepsilon}, v_{\varepsilon}\right) \in\left[L^{\infty}\left(0, T ; V \cap H^{2}(0, \ell)\right)\right]^{2} \\
& \left(u_{\varepsilon}^{\prime}, v_{\varepsilon}^{\prime}\right) \in\left[L^{\infty}(0, T ; V)\right]^{2} \\
& \left(u_{\varepsilon}^{\prime \prime}, v_{\varepsilon}^{\prime \prime}\right) \in\left[L^{\infty}\left(0, T ; L^{2}(0, \ell)\right)\right]^{2} \\
& \left(u_{\varepsilon}^{\prime \prime}, z\right)+\left(v_{\varepsilon}^{\prime \prime}, w\right)+\left(\left(u_{\varepsilon}, z\right)\right)+\left(u_{v^{\prime}}, v_{\varepsilon x}, z+w_{x}\right)+\gamma u_{\varepsilon}^{\prime}(\ell, t) z(\ell)+\alpha\left(v_{\varepsilon}^{\prime}, z\right)+ \\
& \frac{1}{\varepsilon}\left[v_{\varepsilon}(\ell, t)-\phi(\ell)\right]^{-} w(\ell)+\varepsilon v_{\varepsilon}^{\prime}(\ell, t) w(\ell)=(f, z)+(g, w) ; \forall(z, w) \in V^{2}
\end{aligned}
$$

y las condiciones iniciales

$$
\mid \begin{aligned}
& u_{\varepsilon}(\cdot, 0)=u_{0}, u_{\varepsilon}^{\prime}(\cdot, 0)=u_{1} \\
& v_{\varepsilon}(\cdot, 0)=v_{0}, v_{\varepsilon}^{\prime}(\cdot, 0)=v_{1}
\end{aligned}
$$

Demostración. Empleando el método estándar de Faedo-Galerkin, obtenemos el resultado del teorema 1.

\section{Problema de contacto}

En esta sección desarrollaremos la obtención de la solución para el sistema (1). Primeramente daremos una definición de solución para el problema de contacto del sistema Mindlin-Timoshenko.

Definición 3.1 Dado $\left(u_{0}, u_{1}, v_{0}, v_{1}\right) \in V \times(0, \ell) \times \mathbb{K} \times L^{2}(0, \ell)$ y $f, g \in L^{2}(Q)$, diremos que $(u, v)$ es una solución del sistema de contacto (1) si,

$$
\begin{aligned}
& \left(u, u^{\prime}\right) \in L^{\infty}(0, T ; V) \times L^{\infty}\left(0, T ; L^{2}(0, \ell)\right) \\
& \left(v, v^{\prime}\right) \in L^{\infty}(0, T ; V) \times L^{\infty}\left(0, T ; L^{2}(0, \ell)\right), v(t) \in \mathbb{K}
\end{aligned}
$$

satisfaciendo las condiciones iniciales

$$
u(\cdot, 0)=u_{0}, u^{\prime}(\cdot, 0)=u_{1}, v(\cdot, 0)=v_{0}, v^{\prime}(\cdot, 0)=v_{1} \text { en }(0, \ell)
$$

La ecuación

$$
\begin{aligned}
& \left\langle u^{\prime}(\cdot, T), \psi(\cdot, T)-u(\cdot, T)\right\rangle-\left(u_{1}, \psi(\cdot, 0)-u_{0}\right)_{2}-\int_{0}^{T}\left(u^{\prime}, \psi^{\prime}-u^{\prime}\right)_{2} d t+ \\
& \alpha \int_{0}^{T}\left(v^{\prime}, \psi-u\right)_{2} d t+\int_{0}^{T}\left(u_{x}, \psi_{x}-u_{x}\right)_{2} d t+\int_{0}^{T}\left(u+v_{x}, \psi-u\right)_{2} d t=\int_{0}^{T}(f, \psi-u)_{2} d t
\end{aligned}
$$

Para todo $\psi \in H^{1}(0, T ; V)$, y la desigualdad

$$
\begin{aligned}
& \left\langle v^{\prime}(\cdot, T), \xi(\cdot, T)-v(\cdot, T)\right\rangle-\left(v_{1}, \xi(\cdot, 0)-v_{0}\right)_{2}- \\
& \qquad \int_{0}^{T}\left(v^{\prime}, \xi^{\prime}-v^{\prime}\right)_{2} d t+\int_{0}^{T}\left(u+v_{x}, \xi_{x}-v_{x}\right)_{2} d t \geq \int_{0}^{T}(g, \xi-v)_{2} d t .
\end{aligned}
$$

Para todo $\xi \in H^{1}(0, T ; V)$ con $\xi(t) \in \mathbb{K}$, casi siempre en $(0, T)$, donde $\langle\cdot, \cdot\rangle$ representa la dualidad entre $H^{-r}(0, \ell)$ y $H^{r}(0, \ell)$ para algún $r>0$.

Observación: La definición dada representa la solución débil del problema (1). Asumamos que la solución $(u, v)$ de $(1)$, recordando la definición precedente, posee la regularidad $u, v \in$ $L^{2}\left(0, T ; V \cap H^{2}(0, \ell)\right)$ podemos justificar esta definición probando que $(u, v)$ satisface la ecuación de Mindlin-Timoshenko y las condiciones de frontera del sistema (1).

En efecto, tomemos $\xi=u \pm \varphi$, con $\varphi \in C_{0}^{\infty}(Q)$, en (13) e integrando por partes obtenemos la ecuación

$$
\int_{0}^{T}\left(u^{\prime \prime}, \varphi\right)_{2} d t-\int_{0}^{T}\left(\left(u+v_{x}\right)_{x}, \varphi\right)_{2} d t=\int_{0}^{T}(g, \varphi)_{2} d t
$$


que se tiene, por resultado de densidad, para todo $\varphi \in L^{2}(Q)$. Obtener la condición de frontera, Consideramos $\xi=v+\zeta(x) \rho(t)$ en (13) y, usando integración por partes, obtenemos

$$
\begin{aligned}
\int_{0}^{T}\left(v^{\prime \prime}, \zeta(x) \rho(t)\right)_{2} d t-\int_{0}^{T}\left(\left(u+v_{x}\right)_{x}, \zeta(x) \rho(t)\right)_{2} d t+ \\
\int_{0}^{T}\left(u(\ell, t)+v_{x}(\ell, t)\right) \zeta(\ell) \rho(t) d t \geq \int_{0}^{T}(g, \zeta(\ell) \rho(t))_{2} d t
\end{aligned}
$$

Por otro lado, tomando $\varphi=\zeta(x) \rho(t)$ en (14) y combinando el resultado con (15), obtenemos

$$
\int_{0}^{T}\left(u(\ell, t)+v_{x}(\ell, t)\right) \zeta(\ell) \rho(t) d t \geq 0
$$

Para toda función no negativa $\zeta \in C^{1}([0,1])$ y $\rho \in C_{0}^{1}(0, T)$. Así concluimos que

$$
u(\ell, t)+v_{x}(\ell, t) \geq 0, \text { c.s. en }(0, T)
$$

Ahora, escogemos $0 \leq \zeta(x) \leq 1,-1 \leq \rho(t) \leq 1$ para todo $(x, t) \in Q$ y tomando $\xi=$ $v+\zeta(x) \rho(t)(v-\psi)$ en (13) podemos obtener usando integración por partes,

$$
\int_{0}^{T}\left[u(\ell, t)+v_{x}(\ell, t)\right][v(\ell, t)-\phi(\ell)] \zeta(\ell) \rho(t) d t=0
$$

esto implica, por el lema de Dubois-Raymond

$$
\left[u(\ell, t)+v_{x}(\ell, t)\right][v(\ell, t)-\phi(\ell)]=0, \text { c.s. en }[0, T]
$$

La condición de frontera sobre $u$ se sigue análogamente, es decir,

$$
u_{x}(\ell, t)+\gamma u^{\prime}(\ell, t)=0 .
$$

Pasaremos a resolver el problema de contacto para el sistema Mindlin-Timoshenko como límite cuando $\varepsilon \rightarrow 0$ del problema penalizado. Antes de establecer el teorema de existencia de solución débil para (1), necesitamos propiedades previas para obtener lo deseado.

Proposición 3.2 Sea $u_{\varepsilon}, v_{\varepsilon} \in H^{1}(Q)$ satisfaciendo las siguientes propiedades

$$
\begin{aligned}
& u_{\varepsilon}^{\prime \prime}, v_{\varepsilon}^{\prime \prime \prime} \in L^{2}(Q), \quad u_{\varepsilon} \rightarrow u \text { débil en } H^{1}(Q), \quad v_{\varepsilon} \rightarrow v \text { débil en } H^{1}(Q) \\
& \int_{0}^{t}\left|u_{\varepsilon}^{\prime \prime}-u_{\varepsilon x x}\right|_{2}^{2} d t \leq C, \forall \varepsilon>0 \quad y \quad \int_{0}^{t}\left|v_{\varepsilon}^{\prime \prime}-\left(u_{\varepsilon}+v_{\varepsilon x}\right)\right|_{2}^{2} d t \leq C ; \forall \varepsilon>0 .
\end{aligned}
$$

Entonces para $\delta<\frac{1}{2}$

$$
\begin{gathered}
\int_{0}^{t} \int_{(0, \delta) \cup(1-\delta,)}\left(\left|u_{\varepsilon}^{\prime}\right|^{2}-\left|u_{\varepsilon x}\right|^{2}\right) d x d t \leq C \delta \\
\int_{0}^{t} \int_{(0, \delta) \cup(1-\delta,)}\left(\left|v_{\varepsilon}^{\prime}\right|^{2}-\left|u_{\varepsilon x}+v_{\varepsilon x}\right|^{2}\right) d x d t \leq C \delta \\
\lim _{\varepsilon \rightarrow 0} \int_{0}^{t}\left(\left|v_{\varepsilon}^{\prime}\right|_{2}^{2}-\left|u_{\varepsilon x}\right|_{2}^{2}\right) d t=\int_{0}^{t}\left(\left|u^{\prime}\right|_{2}^{2}-\left|u_{x}\right|_{2}^{2}\right) d t \\
\lim _{\varepsilon \rightarrow 0} \int_{0}^{t}\left(\left|v_{\varepsilon}^{\prime}\right|_{2}^{2}-\left|u_{\varepsilon}+v_{\varepsilon x}\right|_{2}^{2}\right) d t=\int_{0}^{t}\left(\left|v^{\prime}\right|_{2}^{2}-\left|u+v_{x}\right|_{2}^{2}\right) d t
\end{gathered}
$$

Demostración. Considerando $z=u_{\varepsilon}^{\prime}(t)$ y $w=v_{\varepsilon}^{\prime}(t)$ en $(7)$ procediendo como en el Teorema 2.1, obtenemos estimativas apropiadas que luego de aplicar el Corolario 4.3 de Dacorogna [2], establecemos el resultado de la existencia de soluciones para el problema de contacto asociado con el sistema de Mindlin-Timoshenko.

Teorema 3.3 Sean $\left(u_{0}, u_{1}, v_{0}, v_{1}\right) \in V \times L^{2}(0,) \times \mathbb{K} \times L^{2}(0, l)$ y $f, g \in L^{2}(Q)$, entonces existe al menos una solución débil del problema (1), de acuerdo a la definición 3.1. 
Demostración. Consideremos los datos $\left(u_{0 \varepsilon}, u_{1 \varepsilon}, v_{0 \varepsilon}, v_{1 \varepsilon}, f_{\varepsilon}, g_{\varepsilon}, \phi\right)$ procediendo como para la obtención del teorema 2.1 se tiene

$$
\left(u_{0 \varepsilon}, u_{1 \varepsilon}, v_{0 \varepsilon}, v_{1 \varepsilon}\right) \rightarrow\left(u_{0}, u_{1}, v_{0}, v_{1}\right) \text { fuerte en } V \times L^{2}(0, \ell) \times \mathbb{K} \times L^{2}(0, \ell)
$$

$\mathrm{y}$

$$
\left(f_{\varepsilon}, g_{\varepsilon}\right) \rightarrow(f, g) \text { fuerte en }\left(L^{2}(Q)\right)^{2}
$$

Tomando $z=\psi-u_{\varepsilon}, w=\xi-v_{\varepsilon}$ con $\psi, \xi \in H^{1}(0, T ; V)$ y $\xi(t) \in \mathbb{K}$ c.s. en $(0, T)$ en el problema penalizado (7) e integrando de 0 hasta $T$, obtenemos,

$$
\begin{gathered}
\left\langle u_{\varepsilon}^{\prime}(\cdot, T), \psi(\cdot, T)-u_{\varepsilon}(\cdot, T)\right\rangle-\left(u_{1 \varepsilon}, \psi(\cdot, 0)-u_{0 \varepsilon}\right)_{2}-\int_{0}^{T}\left(u_{\varepsilon}^{\prime}, \psi^{\prime}-u_{\varepsilon}^{\prime}\right)_{2} d t+ \\
\left\langle v_{\varepsilon}^{\prime}(\cdot, T), \xi(\cdot, T)-v_{\varepsilon}(\cdot, t)\right\rangle-\left(v_{1 \varepsilon}, \xi(\cdot, 0)-v_{0 \varepsilon}\right)_{2}-\int_{0}^{T}\left(v_{\varepsilon}^{\prime}, \xi^{\prime}-v_{\varepsilon}^{\prime}\right)_{2} d t+ \\
\int_{0}^{T}\left(u_{\varepsilon x}, \psi_{x}-u_{\varepsilon x}\right)_{2} d t+\int_{0}^{T}\left(u_{\varepsilon}+v_{\varepsilon x}, \psi-u_{\varepsilon}\right)_{2} d t+\int_{0}^{T}\left(u_{\varepsilon}^{\prime}+v_{\varepsilon x}, \xi_{x}-v_{\varepsilon x}\right)_{2} d t+ \\
\gamma \int_{0}^{T} u_{\varepsilon}^{\prime}(\ell, t)\left(\psi(\ell, t)-u_{\varepsilon}(\ell, t)\right) d t+\alpha \int_{0}^{T}\left(v_{\varepsilon}^{\prime}, \psi-u_{\varepsilon}\right)_{2} d t \\
\begin{aligned}
\frac{1}{\varepsilon} \int_{0}^{T}\left[v_{\varepsilon}^{\prime}(\ell, t)-\phi(\ell)\right]^{-1}\left(\xi(\ell, t)-v_{\varepsilon}(\ell, t)\right) d t & +\xi \int_{0}^{T} v_{\varepsilon}^{\prime}(\ell, t)\left(\xi(\ell, t)-v_{\varepsilon}(\ell, t)\right) d t \\
& =\int_{0}^{T}\left(f_{\varepsilon}, \xi-u_{\varepsilon}\right)_{2} d t+\int_{0}^{T}\left(g_{\varepsilon}, \xi-v_{\varepsilon}\right)_{2} d t
\end{aligned}
\end{gathered}
$$

donde $\langle\cdot, \cdot\rangle$ representa la dualidad entre $H^{-r}(0, \ell)$ y $H^{r}(0, \ell)$ para algún $r>0$. Pasando el límite en (20), cuando $\varepsilon \rightarrow 0$, obtenemos (12) y (13). Para esto, analizamos la convergencia de cada término de (20), cuando $\varepsilon \rightarrow 0$. En efecto, tomando $z=u_{\varepsilon}^{\prime}(t)$ y $w=v_{\varepsilon}^{\prime}(t)$ en (7) y usando el mismo argumento aplicado en el teorema 1 obtenemos la estimativa siguiente

$$
\left|u_{\varepsilon}^{\prime}\right|_{2}^{2}+\left|v_{\varepsilon}^{\prime}\right|_{2}^{2}+\left\|u_{\varepsilon}\right\|_{2}^{2}+\left|u_{\varepsilon}+v_{\varepsilon x}\right|_{2}^{2}+\frac{1}{\varepsilon}\left|\left[v_{\varepsilon}(\ell, t)-\phi(\ell)\right]^{-}\right|^{2}+\gamma \int_{0}^{t}\left|u_{\varepsilon}^{\prime}(\ell, t)\right|^{2} d s+\varepsilon \int_{0}^{t}\left|v_{\varepsilon}^{\prime}(\ell, s)\right|^{2} d s \leq C
$$

Con $C$ una constante positiva. De este resultado garantizamos la existencia de una subsucesión de $\left(u_{\varepsilon}, v_{\varepsilon}\right)$, denotado de la misma forma, tal que

$$
\begin{aligned}
& \left(u_{\varepsilon}, v_{\varepsilon}\right) \rightarrow(u, v) \text { débil } * \text { en }\left[L^{\infty}(0, T ; V)\right]^{2} \\
& \left(u_{\varepsilon}^{\prime}, v_{\varepsilon}^{\prime}\right) \rightarrow\left(u^{\prime}, v^{\prime}\right) \text { débil } * \text { en }\left[L^{\infty}\left(0, T ; L^{2}(0, \ell)\right)\right]^{2} \\
& \left(u_{\varepsilon}^{\prime \prime}, v_{\varepsilon}^{\prime \prime}\right) \rightarrow\left(u^{\prime \prime}, v^{\prime \prime}\right) \text { débil } * \text { en }\left[L^{\infty}\left(0, T ; V^{\prime}\right)\right]^{2}
\end{aligned}
$$

De la convergencia (21)-(23) y Lema 1.4 en [10], obtenemos

$$
\left\{\begin{array}{l}
\left(u_{\varepsilon}, v_{\varepsilon}\right) \rightarrow(u, v) \text { fuertemente en }\left[C\left([0, T] ; H^{\alpha}(0, \ell)\right)\right]^{2} \\
\left(u_{\varepsilon}^{\prime}, v_{\varepsilon}^{\prime}\right) \rightarrow\left(u^{\prime}, v^{\prime}\right) \text { fuertemente en }\left[C\left([0, T] ; H^{-r}(0, \ell)\right)\right]^{2}
\end{array}\right.
$$

donde $\alpha<\frac{1}{2}$ y $r>0$. Por lo tanto, es suficiente escoger $\alpha=r<\frac{1}{2}$ y $r>0$ y de (24) obtener, $\left(u_{\varepsilon}(\cdot, T), v_{\varepsilon}(\cdot, T)\right) \rightarrow(u(\cdot, T), v(\cdot, T))$ fuertemente en $\left[H^{r}(0, \ell)\right]^{2}, \quad \mathrm{y}$ $\left(u_{\varepsilon}^{\prime}(\cdot, T), v_{\varepsilon}^{\prime}(\cdot, T)\right) \rightarrow\left(u^{\prime}(\cdot, T), v^{\prime}(\cdot, T)\right)$ fuertemente en $\left[H^{-r}(0, \ell)\right]^{2}$.

Así concluimos que $\left\langle u_{\varepsilon}^{\prime}(\cdot, T), \psi(\cdot, T)-u_{\varepsilon}(\cdot, T)\right\rangle_{H^{-r} \times H^{r}} \rightarrow\left\langle u^{\prime}(\cdot, T), \psi(\cdot, T)-u(\cdot, T)\right\rangle_{H^{-r} \times H^{r}} \quad$ y

$$
\left\langle v_{\varepsilon}^{\prime}(\cdot, T), \zeta(\cdot, T)-u_{\varepsilon}(\cdot, T)\right\rangle_{H^{-r} \times H^{r}} \rightarrow\left\langle v^{\prime}(\cdot, T), \zeta(\cdot, T)-v(\cdot, T)\right\rangle_{H^{-r} \times H^{r}}
$$

También se tiene que $u_{\varepsilon}$ converge débilmente en $H^{1}(Q)$, lo que implica que $u_{\varepsilon} \rightarrow u$ fuerte en $C\left([0, T] ; H^{\beta}(0, \ell)\right), \beta<1$. Escogemos $\beta>\frac{1}{2}$, obtenemos

$$
u_{\varepsilon}(\ell, 0) \rightarrow u(\ell, 0) \text { en } C([0, T])
$$

puesto que en ese caso: $H^{\beta}(0, \ell) \subset C([0, \ell])$. Podemos obtener resultados similares para $v_{\varepsilon}$. Consideremos ahora la siguiente igualdad (uno de los términos de (20))

$$
\begin{aligned}
-\int_{0}^{T} \int_{0}^{\ell} v_{\varepsilon}^{\prime}\left(\xi^{\prime}-v_{\varepsilon}^{\prime}\right) d x d t+ & \int_{0}^{T} \int_{0}^{\ell}\left(u_{\varepsilon}+v_{\varepsilon x}\right)\left(\psi+\xi_{x}-u_{\varepsilon}-v_{\varepsilon x}\right) d x d t \\
& \int_{0}^{T}\left(\left|v_{\varepsilon}^{\prime}\right|_{2}^{2}-\left|u_{\varepsilon}+v_{\varepsilon x}\right|_{2}^{2}\right) d t+\int_{0}^{T} \int_{0}^{\ell}\left[-v_{\varepsilon}^{\prime} \xi^{\prime}+\left(u_{\varepsilon}+v_{\varepsilon x}\right)\left(\psi+\xi_{x}\right)\right] d x d t
\end{aligned}
$$


y este término por la proposición 1 , converge a

$$
\int_{0}^{T}\left(\left|v^{\prime}\right|_{2}^{2}-\left|u+v_{x}\right|_{2}^{2}\right) d t+\int_{0}^{T} \int_{0}^{\ell}\left[-v_{\varepsilon}^{\prime} \xi^{\prime}+\left(u+v_{x}\right)\left(\psi+\xi_{x}\right)\right] d x d t
$$

y a su vez esto es igual a

$$
-\int_{0}^{T} \int_{0}^{\ell} v^{\prime}\left(\xi^{\prime}-v^{\prime}\right) d x d t+\int_{0}^{T} \int_{0}^{\ell}\left(u+v_{x}\right)\left(\psi+\xi_{x}-u-v_{x}\right) d x d t .
$$

Veamos ahora la siguiente igualdad (otro término de (20))

$$
\begin{aligned}
\frac{1}{\varepsilon} \int_{0}^{T}\left[v_{\varepsilon}(\ell, t)-\phi(\ell)\right]^{-}\left(\xi(\ell)-v_{\varepsilon}(\ell, t)\right) d t=\frac{1}{\varepsilon} \int_{0}^{T}\left(\left[v_{\varepsilon}(\ell, t)-\phi(\ell)\right]^{-}\right)^{2} d t+ & \\
& \frac{1}{\varepsilon} \int_{0}^{T}\left(u_{\varepsilon}(\ell, t)-\phi(\ell)\right)^{-}(\xi(\ell)-\phi(\ell)) d t \geq 0
\end{aligned}
$$

porque $\xi(\ell) \geq \phi(\ell) ; \xi \in \mathbb{K}$. Ahora todos los resultados obtenidos anteriormente, pasando al límite cuando $\varepsilon \rightarrow 0$, llevando a la expresión (20), obtenemos

$$
\begin{gathered}
\left\langle u^{\prime}(\cdot, T), \psi(\cdot, T)-u(\cdot, T)\right\rangle-\left(u_{1}, \psi(\cdot, 0)-u_{0}\right)^{2}-\int_{0}^{T}\left(u^{\prime}, \psi^{\prime}-u^{\prime}\right)_{2} d t+ \\
\left\langle v^{\prime}(\cdot, T), \xi(\cdot, T)-v(\cdot, T)\right\rangle-\left(v_{1}, \xi(\cdot, 0)-v_{0}\right)_{2}-\int_{0}^{T}\left(v^{\prime}, \xi^{\prime}-v^{\prime}\right)_{2} d t+ \\
\int_{0}^{T}\left(u_{x}, \psi_{x}-u_{x}\right)_{2} d t+\int_{0}^{T}\left(u+v_{x}, \psi-u\right)_{2} d t+\int_{0}^{T}\left(u+v_{x}, \xi_{x}-v_{x}\right)_{2} d t+ \\
\gamma \int_{0}^{T}\left[u^{\prime}(\ell, t)(\psi(\ell, t)-u(\ell, t))\right] d t+\alpha \int_{0}^{T}\left(v^{\prime}, \psi-u\right)_{2} d t \geq \int_{0}^{T}(f, \psi-u)_{2} d t+\int_{0}^{T}(g, \xi-v)_{2} d t
\end{gathered}
$$

Para todo $\psi \in H^{1}(Q)$ y $\xi \in H^{1}(Q), \xi \in \mathbb{K}$. Escogeremos ahora $\xi=v$ en (25), obtendremos

$$
\begin{aligned}
& \left\langle u^{\prime}(\cdot, t), \psi(\cdot, t)-u(\cdot, t)\right\rangle-\left(u_{1}, \psi(\cdot, 0)-u_{0}\right)_{2}-\int_{0}^{T}\left(u^{\prime}, \psi^{\prime}-u^{\prime}\right)_{2} d t+ \\
& \int_{0}^{T}\left(u_{x}, \psi_{x}-u_{x}\right)_{2} d t+\int_{0}^{T}\left(u+v_{x}, \psi-u\right)_{2} d t+\gamma \int_{0}^{T}\left[u^{\prime}(\ell, t)(\psi(\ell, t)-u(\ell, t))\right] d t+ \\
& \alpha \int_{0}^{T}\left(v^{\prime}, \psi-u\right)_{2} d t \geq \int_{0}^{T}(f, \psi-u)_{2} d t .
\end{aligned}
$$

Sustituyendo $\psi$ por $-w+2 u$ en 26 , obtenemos:

$$
\begin{aligned}
& \left\langle u^{\prime}(\cdot, t), w(\cdot, t)-u(\cdot, t)\right\rangle-\left(u_{1}, w(\cdot, 0)-u_{0}\right)_{2}-\int_{0}^{T}\left(u^{\prime}, w^{\prime}-u^{\prime}\right)_{2} d t+ \\
& \int_{0}^{T}\left(u_{x}, w_{x}-u_{x}\right)_{2} d t+\int_{0}^{T}\left(u+v_{x}, w-u\right)_{2} d t+\gamma \int_{0}^{T}\left[u^{\prime}(\ell, t)(w(\ell, t)-u(\ell, t))\right] d t+ \\
& \alpha \int_{0}^{T}\left(v^{\prime}, w-u\right)_{2} d t \leq \int_{0}^{T}(f, w-u)_{2} d t .
\end{aligned}
$$

Combinando (26) y (27) para $\psi=w$ obtenemos:

$$
\begin{gathered}
\left\langle u^{\prime}(\cdot, t), \psi(\cdot, t)-u(\cdot, t)\right\rangle-\left(u_{1}, \psi(\cdot, 0)-u_{0}\right)_{2}-\int_{0}^{T}\left(u^{\prime}, \psi^{\prime}-u^{\prime}\right)_{2} d t+ \\
\int_{0}^{T}\left(u_{x}, \psi_{x}-u_{x}\right)_{2} d t+\int_{0}^{T}\left(u+v_{x}, \psi-u\right)_{2} d t+\gamma \int_{0}^{T}\left[u^{\prime}(\ell, t)(\psi(\ell, t)-u(\ell, t))\right] d t+ \\
\alpha \int_{0}^{T}\left(v^{\prime}, \psi-u\right)_{2} d t=\int_{0}^{T}(f, \psi-u)_{2} d t .
\end{gathered}
$$


con lo cual se comprueba la igualdad dada en (12). Finalmente escogemos $\psi=u$ en (25), obtenemos la desigualdad dada en (13), esto es,

$$
\begin{aligned}
\left\langle v^{\prime}(\cdot, t), \xi(\cdot, t)-v(\cdot, t)\right\rangle-\left(v_{1}, \xi(\cdot, 0)-v_{0}\right)_{2}-\int_{0}^{T}\left(v^{\prime}, \xi^{\prime}-v^{\prime}\right) d t+ & \\
& \int_{0}^{T}\left(u+v_{x}, \xi_{x}-v_{x}\right)_{2} d t \geq \int_{0}^{T}(g, \xi-v)_{2} d t
\end{aligned}
$$

y esto completa la prueba.

\section{Comportamiento Asintótico}

Estudiaremos el decaimiento exponencial para la energía $E(t)$ asociado con la solución del problema.

$$
\mid \begin{aligned}
& u^{\prime \prime}-u_{x x}+\left(u+v_{x}\right)=0 \text { en } Q=(0, \ell) \times(0, T) \\
& v^{\prime \prime}-\left(u+v_{x}\right)_{x}+\alpha v^{\prime}=0 \text { en } Q=(0, \ell) \times(0, T) \\
& u(0, \cdot)=v(0, \cdot)=0 \text { sobre }(0, T) \\
& u_{x}(\ell, \cdot)+\gamma u^{\prime}(\ell, \cdot)=0 \text { sobre }(0, T) \\
& u(\ell, \cdot)+v_{x}(\ell, \cdot) \geq 0, v(\ell, \cdot) \geq \phi(\ell) \text { sobre }(0, T) \\
& {\left[u(\ell, \cdot)+v_{x}(\ell, \cdot)\right][v(\ell, \cdot)-\phi(\ell)]=0 \text { sobre }(0, T)} \\
& u(\cdot, 0)=u_{0} ; u^{\prime}(\cdot, 0)=u_{1} ; v(\cdot, 0)=v_{0} ; v^{p}(\cdot, 0)=v_{1} \text { en }(0, \ell)
\end{aligned}
$$

donde $\alpha>0$ es un número real. Esta energía es dada por

$$
E(t)=\frac{1}{2}\left[\left|u^{\prime}(t)\right|_{2}^{2}+\left|v^{\prime}(t)\right|_{2}^{2}+\|u(t)\|^{2}+\left|u(t)+v_{x}(t)\right|_{2}^{2}\right]
$$

Análogamente como en la solución anterior, para datos

$$
\left(u_{0}, u_{1}, v_{0}, v_{1}, \phi\right) \in V \times L^{2}(0, \ell) \times \mathbb{K} \times L^{2}(0, \ell) \times C^{\infty}([0, \ell])
$$

podemos garantizar la existencia de una solución para el problema (28) en la clase

$$
\begin{aligned}
& \left(u, u^{\prime}\right) \in L^{\infty}(0, \infty ; V) \times L^{\infty}\left(0, \infty ; L^{2}(0, \ell)\right) \\
& \left(v, v^{\prime}\right) \in L^{\infty}(0, \infty ; V) \times L^{\infty}\left(0, \infty ; L^{2}(0, \ell)\right), v(t) \in \mathbb{K}
\end{aligned}
$$

Para tratar el decaimiento de la energía (29) del problema (28), primeramente consideramos el problema penalizado

$$
\mid \begin{aligned}
& u_{\varepsilon}^{\prime \prime}-u_{\varepsilon x x}+\left(u_{\varepsilon}+v_{\varepsilon x}\right)=0 \text { en } Q \\
& v_{\varepsilon}^{\prime \prime}-\left(u_{\varepsilon}+v_{\varepsilon x}\right)_{x}+\alpha v_{\varepsilon}^{\prime}=0 \text { en } Q \\
& u_{\varepsilon}(0, \cdot)=v_{\varepsilon}(0, \cdot)=0 \text { sobre }(0, T) \\
& u_{\varepsilon x}(\ell, \cdot)+\gamma u_{\varepsilon}^{\prime}(\ell, \cdot)=0 \text { sobre }(0, T) \\
& u_{\varepsilon}(\ell, \cdot)+v_{\varepsilon x}(\ell, \cdot)-\frac{1}{\varepsilon}\left[v_{\varepsilon}(\ell, \cdot)-\phi(\ell)\right]^{-1} \varepsilon v_{\varepsilon}^{\prime}(\ell, \cdot)=0 \text { sobre }(0, T) \\
& u_{\varepsilon}(\cdot, 0)=u_{0} ; u_{\varepsilon}^{\prime}(\cdot, 0)=u_{1} ; v_{\varepsilon}(\cdot, 0)=v_{0} ; v_{\varepsilon}^{\prime}(\cdot, 0)=v_{1} \text { en }(0, \ell)
\end{aligned}
$$

podemos usar los mismos argumentos como en el problema penalizado (2) para garantizar la existencia y unicidad de la solución para el problema (32) en la clase

$$
\left(u_{\varepsilon}, u_{\varepsilon}^{\prime}, v_{\varepsilon}, v_{\varepsilon}^{\prime}\right) \in L^{\infty}[0, \infty ; V]^{4}
$$

Por dato en la condición del Teorema 1 ( $\operatorname{con} f=g=0$ ). La energía de ese sistema es dada por

$$
E_{\varepsilon}(t)=\frac{1}{2}\left\{\left|u_{\varepsilon}^{\prime}(t)\right|_{2}^{2}+\left|v_{\varepsilon}^{\prime}(t)\right|_{2}^{2}+\left\|u_{\varepsilon}(t)\right\|^{2}+\left|u_{\varepsilon}+v_{\varepsilon x}\right|_{2}^{2}+\frac{1}{\varepsilon}\left|\left[v_{\varepsilon}(\ell, t)-\phi(\ell)\right]^{-}\right|^{2}\right\} .
$$

Tenemos planteado el resultado central por el Teorema siguiente.

Teorema 4.1 Sea $\left(u_{0}, u_{1}, v_{0}, v_{1}, \phi\right) \in V \times L^{2}(0, \ell) \times \mathbb{K} \times L^{2}(0, \ell) \times C^{\infty}([0, \ell])$ con $v_{0}$ satisfaciendo (3) y $\phi(\ell) \geq 0$; entonces existe una constante $w>0$, tal que la energía (29) satisface

$$
E(t) \leq 4 E(0) e^{-w t}, \forall t \geq 0
$$


Demostración. Tomando el producto interno en $L^{2}(0, \ell)$ de $(32)_{1}$, con $u_{\varepsilon}^{\prime}(t)$ y $(32)_{2}$ con $v_{\varepsilon}^{\prime}(t)$ tenemos

$$
\begin{gathered}
\left(u_{\varepsilon}^{\prime \prime}, u_{\varepsilon}^{\prime}\right)+\left(u_{\varepsilon x}, u_{\varepsilon x}^{\prime}\right)+\left(u_{\varepsilon}+v_{\varepsilon x}, u_{\varepsilon x}^{\prime}\right)+\left(v_{\varepsilon}^{\prime \prime}, v_{\varepsilon}^{\prime}\right)+\left(u_{\varepsilon}+v_{\varepsilon x}, v_{\varepsilon x}^{\prime}\right)+\left(\alpha v_{\varepsilon}^{\prime}, v_{\varepsilon}^{\prime}\right)+ \\
u_{\varepsilon}(\ell) v_{\varepsilon}^{\prime}(\ell)+v_{\varepsilon x}(\ell) v_{\varepsilon}^{\prime}(\ell)+\frac{1}{\varepsilon} \frac{d}{d t}\left[v_{\varepsilon}(\ell, t)-\phi(\ell)\right]^{-1}+\varepsilon v_{\varepsilon}^{\prime}(\ell) v_{\varepsilon}^{\prime}(\ell)- \\
\quad\left(u_{\varepsilon x} \times\left. u_{\varepsilon}^{\prime}\right|_{0} ^{\ell}\right)-\left.\left(u_{\varepsilon}+u_{\varepsilon x}\right) v_{\varepsilon}^{\prime}\right|_{0} ^{\ell}=0
\end{gathered}
$$

de donde usando datos dados en (32) y definición de energía dada en (34), obtenemos la estimativa:

$$
E_{\varepsilon}^{\prime}(t) \leq-\gamma\left\|u_{\varepsilon}^{\prime}(\ell, t)\right\|-\alpha\left|v_{\varepsilon}^{\prime}\right|_{2}^{2}-\varepsilon\left|v_{\varepsilon}^{\prime}(\ell, t)\right|^{2} .
$$

esto es, $E_{\varepsilon}(t)$ es una función no creciente. Para un $\eta>0$ cualquiera, definamos la energía perturbada

$$
E_{\varepsilon \eta}(t)=E_{\varepsilon}(t)+\eta \psi(t)
$$

con $\psi(t)=\theta\left(u_{\varepsilon}^{\prime}(t), u_{\varepsilon}(t)\right)_{2}+\theta\left(v_{\varepsilon}^{\prime}(t), v_{\varepsilon}(t)\right)_{2}+\left(u_{\varepsilon}^{\prime}(t), x u_{\varepsilon x}(t)\right)_{2}$ donde $\theta>0$ que será determinado. Observemos que

$$
|\psi(t)| \leq\left(\frac{1+\theta}{2}\right)\left|u_{\varepsilon}^{\prime}(t)\right|_{2}^{2}+\theta\left|v_{\varepsilon}^{\prime}(t)\right|_{2}^{2}+\left(\frac{1}{2}+\theta\right)\left\|u_{\varepsilon}(t)\right\|^{2}+\frac{\theta}{2}\left|u_{\varepsilon}(t)+v_{\varepsilon x}(t)\right|_{2}^{2}
$$

esto es,

$$
|\psi(t)| \leq 2(1+3 \theta) E_{\varepsilon}(t)
$$

De (35) y (38), obtenemos la equivalencia de las energías de $E_{\varepsilon}(t)$ y $E_{\varepsilon \eta}$ :

$$
\left|E_{\varepsilon \eta}(t)-E_{\varepsilon}(t)\right|=\eta|\psi(t)| \leq 2(1+3 \theta) E_{\varepsilon}(t)
$$

o

$$
[1-2(1+3 \theta) \eta] E_{\varepsilon}(t) \leq E_{\varepsilon \eta}(t) \leq[1+2(1+3 \theta) \eta] E_{\varepsilon}(t) .
$$

Tomando $0<\eta \leq \frac{1}{4(1+3 \theta)}$ y de (39) obtenemos el simplificado

$$
\frac{E_{\varepsilon}(t)}{2} \leq E_{\varepsilon \eta}(t) \leq 2 E_{\varepsilon(t)}
$$

Considerando la derivada de la función $\psi(t)$ y de $(32)_{1}$ y $(32)_{2}($ con $f=g=0)$, obtenemos,

$$
\begin{aligned}
& \psi^{\prime}(t)=\theta\left|u_{\varepsilon}^{\prime}(t)\right|_{2}^{2}+\theta\left|v_{\varepsilon}^{\prime}(t)\right|_{2}^{2}-\theta\left\|u_{\varepsilon}(t)\right\|^{2}-\theta\left|u_{\varepsilon}(t)+v_{\varepsilon}(t)\right|_{2}^{2}- \\
& \frac{\theta}{\varepsilon}[v(\ell)-\theta(\ell)]^{-} v(\ell)-\gamma \theta u_{\varepsilon}^{\prime}(\ell, t) u_{\varepsilon}(\ell, t)-\varepsilon \theta v_{\varepsilon}^{\prime}(\ell, t) v_{\varepsilon}(\ell, t)-\alpha \theta\left(v_{\varepsilon}^{\prime}(t), v_{\varepsilon}(t)\right)_{2}+ \\
& \quad\left(u_{\varepsilon x x}(t), x u_{\varepsilon x}(t)\right)_{2}\left(u_{\varepsilon}(t)+v_{\varepsilon x}(t), x u_{\varepsilon}(t)\right)_{2}+\left(u_{\varepsilon}^{\prime}(t), x u_{\varepsilon x}^{\prime}(t)\right)_{2}
\end{aligned}
$$

Analizaremos los términos que aparecen en el lado derecho de (41):

$$
\begin{aligned}
\left(u_{\varepsilon x x}(t), x u_{\varepsilon x}(t)\right)_{2} & =\frac{1}{2} \int_{0}^{\ell} x \frac{\partial}{\partial x}\left|u_{\varepsilon x}(t)\right|^{2} d x \\
& =-\frac{1}{2} \int_{0}^{\ell}\left|u_{\varepsilon x}(x, t)\right|^{2} d x+\frac{1}{2}\left|u_{\varepsilon x}(\ell, t)\right|^{2} \\
\left(u_{\varepsilon}^{\prime}(t), x u_{\varepsilon x}^{\prime}(t)\right)_{2} & =\frac{1}{2} \int_{0}^{\ell} x \frac{\partial}{\partial x}\left|u_{\varepsilon}^{\prime}(x, t)\right|^{2} d x \\
& =-\frac{1}{2} \int_{0}^{\ell}\left|u_{\varepsilon}^{\prime}(x, t)\right|^{2} d x+\frac{1}{2}\left|u_{\varepsilon}^{\prime}(\ell, t)\right|^{2} \\
-\frac{\theta}{\varepsilon}[v(\ell)-\phi(\ell)]^{-} v(\ell) & =-\frac{\theta}{\varepsilon}\left|[v(\ell)-\phi(\ell)]^{-}\right|^{2}-\frac{\theta}{\varepsilon}[v(\ell)-\phi(\ell)]^{-} \phi(\ell) \\
\leq & -\frac{\theta}{\varepsilon}\left|[v(\ell)-\phi(\ell)]^{-}\right|^{2}
\end{aligned}
$$


Puesto que $\phi(\ell) \geq 0$.

$$
-\gamma \theta u_{\varepsilon}^{\prime}(\ell, t) u_{\varepsilon}(\ell, t) \leq C_{1}\left|u_{\varepsilon}^{\prime}(\ell, t)\right|^{2}+\xi\left\|u_{\varepsilon}(t)\right\|^{2}
$$

donde $C_{1}=\frac{\gamma^{2} \theta^{2} C_{0}^{2}}{\xi}, C_{0}>0$ es la constante tal que $\left|u_{\varepsilon}(\ell, t)\right| \leq C_{0}\left\|u_{\varepsilon}(t)\right\|$ y $\xi>0$ es un número real a elegir.

$$
-\varepsilon \theta v_{\varepsilon}^{\prime}(\ell, t) \varepsilon(\ell, t) \leq C_{2} \varepsilon^{2}\left|v_{\varepsilon}^{\prime}(\ell, t)\right|^{2}+\xi\left|u_{\varepsilon}(t)+v_{\varepsilon x}(t)\right|_{2}^{2}+\xi\left\|u_{\varepsilon}(t)\right\|^{2}
$$

donde $C_{2}=\frac{2 \theta^{2} C_{0}^{2}}{\xi}$

$$
-\alpha \theta\left(v_{\varepsilon}^{\prime}(t), \varepsilon(t)\right) \leq C_{3}\left|v_{\varepsilon}^{\prime}(t)\right|_{2}^{2}+\xi\left|u_{\varepsilon}(t)+v_{\varepsilon x}(t)\right|_{2}^{2}+\xi\left\|u_{\varepsilon}(t)\right\|^{2}
$$

donde $C_{3}=\frac{2 \alpha^{2} \theta^{2}}{\xi}$

$$
-\left(u_{\varepsilon}(t)+v_{\varepsilon x}, x u_{\varepsilon x}(t)\right) \leq \frac{3}{8}\left|u_{\varepsilon}(t)+v_{\varepsilon x}(t)\right|_{2}^{2}+\frac{2}{3}\left\|u_{\varepsilon}(t)\right\|^{2}
$$

Sustituyendo (42)-(48) en (41) y observando (32) $)_{4}$ obtenemos

$$
\begin{gathered}
\psi^{\prime}(t) \leq-\left(\frac{1}{2}-\theta\right)\left|u_{\varepsilon}^{\prime}(t)\right|_{2}^{2}+\left(\theta+C_{3}\right)\left|v_{\varepsilon}^{\prime}(t)\right|_{2}^{2}-\left(\theta-\frac{1}{6}\right)\left\|u_{\varepsilon}(t)\right\|^{2}- \\
\left(\theta-\frac{3}{8}\right)\left|u_{\varepsilon}(t)+v_{\varepsilon x}(t)\right|_{2}^{2}-\frac{\theta}{\varepsilon}\left|[v(\ell)-\phi(\ell)]^{-}\right|^{2}+\frac{1}{2}\left(1+2 C_{1}+\gamma^{2}\right)\left|u_{\varepsilon}^{\prime}(\ell, \cdot)\right|^{2}+ \\
C_{2} \varepsilon^{2}\left|v_{\varepsilon}^{\prime}(\ell, t)\right|^{2}+3 \xi\left\|u_{\varepsilon}(t)\right\|^{2}+2 \xi\left|u_{\varepsilon}(t)+v_{\varepsilon x}(t)\right|_{2}^{2}
\end{gathered}
$$

Por tanto, para $\frac{3}{8}<\theta<\frac{1}{2}$, obtenemos una forma simplificada para $\psi(t)$,

$$
\begin{aligned}
\psi^{\prime}(t) \leq-\rho E_{\varepsilon}(t)+\left(\frac{1}{2}+\theta+C_{3}\right)\left|v_{\varepsilon}^{\prime}(t)\right|_{2}^{2}+ & \\
& \frac{1}{2}\left(1+2 C_{1}+\gamma^{2}\right)\left|u_{\varepsilon}^{\prime}(\ell, \cdot)\right|^{2}+C_{2} \varepsilon^{2}\left|v_{\varepsilon}^{\prime}(\ell, t)\right|^{2}+6 \xi E_{\varepsilon}(t)
\end{aligned}
$$

donde $\rho=\operatorname{mín}\left\{\frac{1}{2}-\theta, \theta-\frac{3}{8}\right\}>0$. Derivando la expresión (35) y observando (36) y (49) se tiene

$$
\begin{aligned}
E_{\varepsilon \eta}^{\prime}(t) \leq-\eta(\rho-6 \xi) E_{\varepsilon}(t)-\left[\alpha-\eta\left(\frac{1}{2}+\theta+C_{3}\right)\right]^{2}\left|v_{\varepsilon}^{\prime}(t)\right|_{2}^{2}- & \\
& {\left[\gamma-\frac{\eta}{2}\left(1+2 C_{1}+\gamma^{2}\right)\right]\left|u_{\varepsilon}^{\prime}(\ell, \cdot)\right|^{2}-\left(\varepsilon-\eta C_{2} \varepsilon^{2}\right)\left|v_{\varepsilon}^{\prime}(\ell, t)\right|^{2} }
\end{aligned}
$$

Tomando $0<\eta \leq \min \left\{\frac{2 \alpha}{1+\theta+C_{3}}, \frac{2 \gamma}{1+2 C_{1}+\gamma^{2}}, \frac{1}{C_{2} \varepsilon}, \frac{1}{4(1+3 \theta)}\right\}$,

$0<\xi<\frac{\rho}{6}$, y recordando (40), obtenemos $E_{\varepsilon \eta}^{\prime}(t)+w E_{\varepsilon \eta}(t) \leq 0$ con $w=\left[\frac{\rho}{2}-2 \xi\right] \eta$. Integrando y aplicando desigualdad de Gronwall

$$
\begin{gathered}
\int_{0}^{t} E_{\varepsilon \eta}^{\prime}(s) d s+w \int_{0}^{t} E_{\varepsilon \eta}(s) d s \leq 0, \text { entonces } E_{\varepsilon \eta}(t) \leq E_{\varepsilon \eta}(0)-\int_{0}^{t} E_{\varepsilon \eta}(s) d s \\
E_{\varepsilon \eta}(t) \leq E_{\varepsilon \eta}(0) e^{-w t}, \forall t \geq 0
\end{gathered}
$$

Combinando (40) y (51) concluimos

$$
E_{\varepsilon}(t) \leq 4 E_{\varepsilon}(0) e^{-w t}, \forall t \geq 0 .
$$

Por tanto, pasando $\lim _{\varepsilon \rightarrow 0}$ ínf en (52), podemos concluir $E(t) \leq 4 E(0) e^{-w t}, \forall t \geq 0$. 


\section{Discusión y conclusiones}

El sistema planteado en (1) es una modificación al planteado por F.D. Araruna [5], agregándose un término apropiado de amortiguamiento interno $\alpha^{\prime} v$ en la primera ecuación, que gobierna el desplazamiento vertical. El problema de la existencia puede ser tratado con $\gamma=0$, pero consideramos $\gamma>0$ con la finalidad de estudiar el comportamiento asintótico de la energía asociada con las soluciones del sistema (1). Podemos concluir que el sistema podría también trabajarse con un término local de amortiguamiento externo o también podría incrementarse una memoria en algunas de las ecuaciones del sistema (1), que nos permitiría informarnos procesos físicos precedentes, como caracterizan a los sistemas viscoelásticos. 


\section{REFERENCIAS BIBLIOGRÁFICAS}

[1] Copetti M.I.M., Elliot, C.M. (1993). A one-dimensional quasi-static contact problem in linear thermoelasticity. European Journal of Applied Mathematics, $4(2): 151-174$.

[2] Dacorogna B. (1982). Weak continuity and weak lower semicontinuity of nonlinear functionals. Lectures Notes in Mathematics. Springer, Berlin.

[3] Duvaut, G. (1969). Probleme di signorini en viscoelasticité linéaire. Comptes Rendus de L'Acadmie des Sciences de Paris, 268:1044-1046.

[4] Duvaut, G., Lions, J. L. (1972). Les Inéquations on Mécanique et en Physique. Dunod, Paris.

[5] Araruna, F. D., Feitosa, A.J. R., Olivera, M. L. (2009). A boundary obstacle problem for the Mindlin-Timoshenko system. Mathematical Methods in the Applied Sciences, 32(6):738-756.

[6] Portillo, H. (1999). Estabilidad para problemas de contacto y materiales mixtos, tesis de doctorado. Instituto de Matemática de la UFRJ-Brail.

[7] Kim J. U. (1989). A boundary thin obstacle problem for a wave equation. Communications in Partial Differential Equations, 14(8-9):1011-1026.

[8] Muñoz, J., Olivera, M. L. (1977). Exponential stability for a contact problem in thermoelasticity. IMA Journal of Applied Mathematics, 58:71-82.

[9] Signorini, A. (1959). Questioni de elasticitá non linearizzata e semilinearizzata. Rendiconti di Matematica e delle sue Applicazioni, 18(5):95.

[10] Signorini A. (1993), Sopra alcune questioni di elastostatica. Atti della Societa per il Progresso della Scienza, 513-533.

[11] Elliot C. M. (1994), A dynamic contact problem in thermoelasticity. Nonlinear Analysis Theory Methods and Applications, 23(7):883-898.

[12] Nakao M., Muñoz Rivera J. (2001), The contact problem in thermoviscoelastic materials. Journal of Mathematical Analysis ans Applications, 264(2) :522-545.

[13] Muñoz Rivera J., Portillo Oquendo H. (2001), Exponential stability to a contact problem of partially viscoelastic materials. Journal of Elasticity, 63(2):87-111. 